\title{
Severe exacerbation of post traumatic syringomyelia after lithotripsy. Case report
}

\author{
N D Lorenzo MD, A Maleci MD PhD, B M Williams MD ChM FRCS \\ Institute of Neurology \& Neurosurgery, Cagliari, Italy and Midland Centre for \\ Neurosurgery and Neurology, Warley, England
}

\begin{abstract}
A case of post traumatic thoracic syringomyelia is reported which presented with an acute and severe worsening of the spinal cord condition after extracorporeal shock wave lithotripsy was performed for ureteral stone. The worsening was probably caused by the shock waves reverberating the fluid within the intramedullary cavity producing further damage to the spinal cord.
\end{abstract}

Keywords: spinal cord injury; syringomyelia; lithotripsy.

\section{Introduction}

In recent years interest has been maintained in the diagnosis, treatment and pathogenesis of syringomyelia, aided by the increasing incidence of this condition revealed by means of magnetic resonance imaging (MRI).

Several pathogenetic hypotheses linked to some derangement of normal cerebrospinal fluid (CSF) flow have been proposed regarding this controversial condition.

In the commonest variety, which is hindbrain related syringomyelia, there is usually a hindbrain hernia blocking the foramen magnum with cerebellar tonsils which are impacted producing a valve like morphology. For such cases many mechanisms have been proposed, including the following:

1 arterial pulsation of the CSF transmitted to the cervical cord ${ }^{1}$

2 differential pressures between the cranium and the spinal canal ${ }^{2}$

3 transmedullary passage of CSF along the Virchow-Robin spaces; ${ }^{3,4}$

Williams $^{2}$ proposed that energy transmitted from the thoracic and abdominal cavities into the spine might be of importance in the pathogenesis of syringomyelia. The valveless veins of the plexus of Batson

Correspondence: N Di Lorenzo MD, Neurochirurgia, Ospedale Marino, Viale Poetto 12, 09100 Cagliari, Italy. surrounding the bones and communicating with the epidural veins are the ideal conduit for such energy. The epidural veins become distended in response to a rise of intraabdominal pressure and compress the dura during such activities as a cough. The result is vigorous movement of the CSF upwards. If there is a syrinx cavity present then fluid may surge impulsively and inflict energy on the inside of the syrinx. This may be called the 'slosh' mechanism. In this way the abrupt worsening that patients often report during or immediately after intense physical efforts such as coughing, sneezing or laborious defecation could be explained.

In the case of post traumatic syringomyelia, the haematomyelia and necrosis forming liquefaction of the haematoma core is thought sometimes to be the cause of this subgroup of syringomyelia cases. ${ }^{5}$

We report a case where an acute and severe deterioration of spinal cord function seems likely to be due to the reverberation, generated by extracorporeal shock waves used for lithotripsy procedure, of the fluid within a thoracic post traumatic syrinx with consequent further damage to the nervous tissue.

\section{Case report}

This 58 year old man with normal habitus ( $171 \mathrm{~cm}$ in height, weighing $78 \mathrm{kgs}$ ), had a traumatic fracture of the body of T12 30 years ago. 
Since then he suffered a neurological deficit in the lower limbs which had stabilised through the years. He could walk for a short distance with canes. Sphincter function was preserved; he complained of hypoaesthesia of the legs, but the exact deficits were not recorded by neurological examination before the procedure. At the age of 55 he had recurrent symptoms of renal colic caused by a calcium oxalate stone in the upper third of the right ureter diagnosed by sonography and urography.

Lithotripsy was undertaken under neuroleptoanalgesia. The power output of the machine used was around $16 \mathrm{kV}$.

Immediately after the procedure he realised that he could no longer move his legs. A neurological examination revealed a flaccid paraplegia with urinary retention and analgesia from T10 downwards; no other unwanted events were encountered and the stone problem was resolved. MRI examination disclosed a discrete T9-12 syringomyelic cavity. The neurology remained substantially unchanged. At present the patient is chair-bound even though minimal movements of the distal part of the legs are possible and some sensation has returned. A new MRI examination (Fig 1) did not show any variation from the first one. Because of the stable clinical and radiological picture the patient has not been offered surgical treatment.

\section{Discussion}

Our case was not satisfactorily studied before the lithotripsy procedure, but it is likely that he developed a syringomyelic cavity after the trauma 30 years previously. The disease was stabilised until the shock waves used to fragment the ureteral stone affected the fluid contained in the syrinx. Extracorporeal lithotripsy is a procedure which employs shock waves to fragment and destroy stones in the kidneys. ${ }^{6,7}$ Water and water-like fluids such as are in syrinx cavities are good transmitters of shock waves, so that the impact has been transmitted in turn to the nervous tissue surrounding the cavity leading to the serious and irreversible deficits that our patient experienced.

It seems that this case supports the idea that energy transmission from the contained fluid to the cord walls is a factor in the evolution of syringomyelic cavities. In the sloshing hypothesis, coughing and sneezing

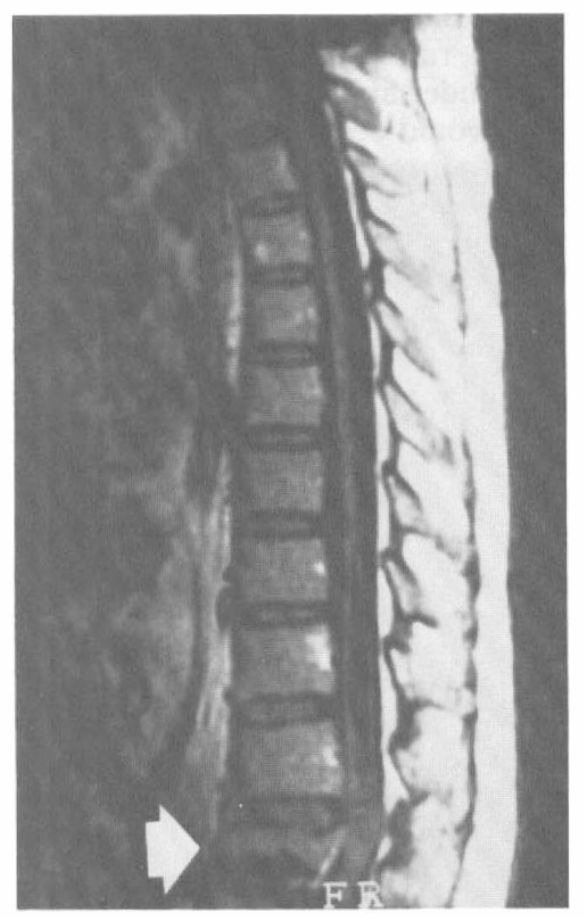

Figure 1 MRI, sagittal view, of the thoracic spine showing a discrete intramedullary cavity at T9-12 level and an old fracture of the body of T12 (arrow).

and physical efforts are responsible for rise of pressure in the syringomyelic cavities and the progressive dissection of the surrounding nervous tissue. The hammering action of lithotripter shock waves is more intense and more focused than that caused by physiological efforts and this may explain why in our case the worsening of neurological deficits has been so acute and severe. It cannot be guessed whether the shock wave affected the syrinx through lateral dispersion of the waves or secondary waves or whether energy was inflicted upon the syrinx through the medium of the veins. The left renal vein in particular is reported as having a wide connection to the spinal veins.

Fluids collections are not present physiologically within the spinal cord, and thus shock wave lithotripsy is normally a safe procedure. If other cases should be found with neurological signs, even minor, pointing toward the possibility of syringomyelia, 
an MRI examination should be obtained in order to rule out intramedullary cavity before undertaking lithotripsy. This precaution would seem to be particularly applicable to incomplete or complete paraplegics who have both a susceptibility to renal stones and an incidence of post traumatic syringomyelia of around $3 \% .^{2}$ It seems likely that damage can occur after litho- tripsy in a previously asymptomatic syrinx and in patients of this sort it might be thought wise to carry out MRI on the fracture site before lithotripsy. The same precautions might be thought relevant in other cases where cord cavities and kidney stones may coexist, including longstanding hindbrain related syringomyelia and spina bifida patients.

\section{References}

1 Gardner WJ (1965) Hydrodynamic mechanism of syringomyelia: Its relationship to myelocele. J Neurol Neurosurg Psychiatry 28: 247-259.

2 Williams B (1993) Pathogenesis of syringomyelia. Acta Neurochir 123: 159-165.

3 Aboulker J (1979) La syringomyélie et les liquids intra-rachiens. Neurochirurgie 25 (Suppl 1): 1-44.

4 Ball BJ, Dayan AD (1972) Pathogenesis of syringomyelia. Lancet 2: 799-801.

5 Kakoulas B (1984) Pathology of spinal injuries. Cent Nerv Syst Trauma 1: 117-129.

6 Homes SAV, Whitfield HN (1991) The current status of lithotripsy. Br J Urol 68: 337-344.

7 Tolley DA, Wallace DMA, Tiptaft RC (1991) First UK Consensus Conference on Lithotriptor Terminology 1989. Br J Urol 67: 9-12. 